\title{
Performance of Coloured Synthetic Broiler Chicken Fed Dried Azolla as Protein Substitute
}

\author{
Subhranshu Sekhar Rout ${ }^{1 *}$, C.R. Pradhan ${ }^{1}$, S.K. Mishra ${ }^{2}$, P.K. Pati ${ }^{3}$ and J. Bagh ${ }^{1}$ \\ ${ }^{1}$ Department of Livestock Production and Management, ${ }^{2}$ Department of Animal Nutrition, \\ ${ }^{3}$ Department of Livestock Products and Technology, College of Veterinary Science and Animal \\ Husbandry, Orissa University of Agriculture and Technology, Bhubaneswar, Odisha, India \\ *Corresponding author
}

\section{A B S T R A C T}

The study was carried out to evaluate the growth performance of colour synthetic broiler chicken fed with sun dried Azolla as protein substitute. The 150 day-old broiler chicks of either sex were distributed randomly in five dietary treatments with 3 replicates each, $\mathrm{C} 1$ : basal diet, T1: basal diet $+10 \%$ dietary protein from Azolla, T2: basal diet $+10 \%$ dietary protein from Azolla + enzyme, T3: basal diet+ 15\% dietary protein from Azolla, T4: basal

\section{Keywords}

Azolla, Growth

Performance,

Coloured Synthetic

Broiler, Enzyme.

Article Info

Accepted:

17 October 2017

Available Online:

10 December 2017 diet $+15 \%$ dietary protein from Azolla + enzyme. The experiment was carried out for 92 days for evaluating performance with respect to body weight, body weight gain, feed consumption, feed conversion ratio (FCR), and economics of production. Each of the Azolla fed group, compared to control, showed significantly $(\mathrm{P} \leq 0.05)$ higher body weight. T4 showed higher gains than $\mathrm{T} 1$ till $4^{\text {th }}$ week whereas no significant $(\mathrm{P} \geq 0.05)$ difference was recorded between the Azolla fed groups from $5^{\text {th }}$ week onwards. Azolla fed group registered significantly $(\mathrm{P} \leq 0.05)$ higher consumptions than the control from $2^{\text {nd }}$ week. However between the two non-enzymes groups $\mathrm{T} 1$ showed higher $(\mathrm{P} \leq 0.05)$ values than T3. Enzyme supplementation resulted in a lower consumption in $10 \%$ Azolla group while it led to a higher consumption in $15 \%$ Azolla group, and that between the two enzyme groups, group $\mathrm{T} 4$ showed higher values $(\mathrm{P} \leq 0.05)$ than group $\mathrm{T} 2$. Throughout the experiment feed efficiency of Azolla fed group showed higher than the control. Cost benefit analysis on basis of net return/bird or net return $/ \mathrm{kg}$ live weight, each of Azolla fed groups showed higher economic efficiency than the control. It was concluded that Azolla could be included in the broiler ration up to $15 \%$ level which resulted in higher body weight gain and feed efficiency. It leads to substantial economisation of feed cost of production.

\section{Introduction}

Poultry is one of the fastest growing segments of the agricultural sector in India today. The growth of country's poultry sector is however, confronted with a major stumbling blockscarcity and high price of feed ingredients. Since feed cost alone accounts for $60-70 \%$ of total production cost Bhuiyan et al., (1998), it is essential that all possible efforts be made for lowering feed cost for a sustainable and rewarding poultry enterprise. The shrinking resources of world grain production and its escalating cost has triggered a search for cheap unconventional feeds for poultry production Shamna et al., (2013). Aquatic 
plant species have been observed to be a fairly cheap feedstuff for poultry and can partly substitute the conventional and expensive dietary protein sources Haustein et al., (1994). Of the various species, the cosmopolitan fern Azolla is perhaps the most promising from the point of view of ease of cultivation, productivity and nutritive value Becerra et al., (1995). Azolla is very rich in proteins, essential amino acids, chlorophyll, vitamins (vitamin A, vitamin B12 and BetaCarotene), growth promoter intermediaries and minerals like calcium, phosphorous, potassium, ferrous, copper, magnesium etc. Azolla could replace $20-25 \%$ of commercial feed and exhibits excellent results in poultry (Subudhi and Singh, 1978). Hence, the proposed study is undertaken to explore the potential effect of supplementation of Azolla on the performance of coloured synthetic broiler birds in terms of body weight gain, feed efficiency, and cost efficiency of production.

\section{Materials and Methods}

The 150 day-old colour synthetic broiler chicks of either sex belonging to a single hatch were procured from poultry farm, College of Veterinary Science \& Animal husbandry and reared in Instructional Livestock Farm in deep litter system of rearing and were distributed randomly in five dietary treatments with 3 replicates each, $\mathrm{C} 1$ : basal diet, T1: basal diet $+10 \%$ dietary protein from Azolla, T2: basal diet $+10 \%$ dietary protein from Azolla + enzyme, T3: basal diet $+15 \%$ dietary protein from Azolla, T4: basal diet $+15 \%$ dietary protein from Azolla + enzyme. For the preparation of experimental diets, Azolla was cultivated in Instructional Livestock Farm, Orissa University of Agriculture and Technology, Bhubaneswar as per the standard procedures Pillai et al., (2002) with slight modification. The diets were made isocaloric and isonitrogenous. The ingredient compositions of the broiler starter and broiler finisher rations, nutrient compositions of the broiler starter and finisher rations are presented in Tables 1 and 2 respectively.

The chemical composition such as DM, CP, ether extract, crude fiber, NFE, total ash and acid insoluble ash, of Azolla samples were analysed as per AOAC (1995). Determination of calcium was done as the method modified by Talapatra et al., (1940). The micro minerals viz. zinc, copper, manganese and iron contents in the samples were estimated in atomic absorption spectrophotometer $\left(\right.$ ELECO-246 $\left.{ }^{\circledR}\right)$. The day old chicks were wing banded for identification. The chicks were brooded by electric brooder with hover for one week. All the chicks were vaccinated against Ranikhet Disease (Lasota strain) on $7^{\text {th }}$ day, Infectious Bursal Disease (Intermediate strain) on $14^{\text {th }}$ day and Ranikhet Disease vaccine (Lasota strain) booster on $28^{\text {th }}$ day. The weekly feed intake and body weight was recorded.

\section{Recording of body weight}

Body weight of $1^{\text {st }}$ week and thereafter at weekly intervals up to $7^{\text {th }}$ week was recorded. The body weight gain was calculated by subtracting the initial body weight from final body weight of the periods and cumulative gains for successive weeks were calculated.

\section{Feed consumption and feed conversion ratio (FCR)}

The group average feed consumption was calculated by subtracting the residual feeds at the end of each week from the total feed provided to the birds during the said weeks. Cumulative feed consumption was calculated by adding from $1^{\text {st }}$ week feed consumption up to the desired week. Feed conversation ratio was derived by dividing cumulative feed 
consumption up to a particular week with cumulative body gain up to that week.

$\mathrm{FCR}=\frac{\text { Cumulative feed consumption }(\mathrm{g}) \text { up to a particular week }}{\text { Cumulative body weight gain }(\mathrm{g}) \text { uptothat week }}$

\section{Economics of broiler production}

The cost of different diet used in the study was worked out based on the prevailing prices of the constituent feed ingredients, minerals, salts and other additives including that of enzymes. The differences in returns between groups were ascribed only to the costs of the diets. The relative cost effectiveness of each diet was thus assessed. The calculated costs of feed for the treatment groups are presented in Table 3 and 4.

\section{Statistical analysis}

The statistical analysis of the data was done according to the method described by (Snedecor and Cochran, 1998). Charts and calculations were done with the help of Data analysis tool of Office 2007.

\section{Chemical composition of Azolla meal}

The chemical analysis of ground dried Azolla used in experimental rations is presented in Table 5.

\section{Weekly body weight and body weight gain}

The average body weights and average cumulative weight gains for the birds at weekly interval up to $7^{\text {th }}$ week of age in different treatment groups are presented in Table 6. No significant $(\mathrm{P} \geq 0.05)$ difference in body weight was observed till the end of $2^{\text {nd }}$ week, while significant $(\mathrm{P} \leq 0.05)$ differences were observed between groups from $3^{\text {rd }}$ week onwards.
Azolla fed group, showed significantly $(\mathrm{P} \leq 0.05)$ higher body weight compared to control from $3^{\text {rd }}$ to $7^{\text {th }}$ week.

The highest and lowest cumulative gains at the end of both starter phase $\left(3^{\text {rd }}\right.$ week) and finisher phase $\left(7^{\text {th }}\right.$ week) were shown by treatments $\mathrm{T} 4$ and $\mathrm{C} 1$, respectively. While no difference $(\mathrm{P} \geq 0.05)$ was noticed between the groups in $2^{\text {nd }}$ week. However significant $(\mathrm{P} \geq 0.05)$ differences were observed between groups from $3^{\text {rd }}$ week onwards.

Azolla fed group, in comparison to the control, registered higher $(\mathrm{P} \leq 0.05)$ weight gains in every week from $3^{\text {rd }}$ to $7^{\text {th }}$ week, except in $7^{\text {th }}$ week when groups $\mathrm{C} 1$ and T1 had no significant $(\mathrm{P} \geq 0.05)$ differences. From these findings it may be interpreted that, Azolla, which is rich in crude protein and contains a high metabolizable energy (ME) level, might have improved digestion and also, might have increased the availability of dietary nutrients as reported by several authors Subudhi and Singh, (1978), Cambel, (1984), Querubin et al.,(1986), Sarria and Preston, (1995), (Basak et al., 2002), Seth et al., (2014), Chichilichi et al., (2014)

Body weights or body weight gains for the enzyme supplemented group, T2 and T4 were not significantly $(\mathrm{p} \geq 0.05)$ different from $\mathrm{T} 1$ and T3, respectively. However numerically higher body weights and weight gains were recorded for groups T2 and T4 in all age groups. This implied that enzyme supplementation with both $10 \%$ and $15 \%$ levels of Azolla inclusion in the diet improved the growth rate of birds. This might be due to improved digestibility of feed due to enzyme supplementation Rajeshwara and Devegowda, (1996) and Choct et al., (1995) stated that suitable enzyme combination strategies from different feed ingredients might result in an increase in feed intake, stimulation of growth and improvement of feed conversion. 


\section{Feed consumption}

The cumulative weekly feed consumptions, for the birds in different dietary treatments, are presented in Table 7. Each of the Azolla fed group registered significantly $(\mathrm{P} \leq 0.05)$ higher consumptions than the control from the $2^{\text {nd }}$ week continuing till the end of the experiment Differences $(\mathrm{p} \leq 0.05)$ were also recorded between the Azolla fed groups except in the $2^{\text {nd }}$ week.

In the non-enzyme groups, in the present study, inclusion of Azolla at 10\% level resulted in increase in feed consumption compared to the controls. This corroborated the findings of Cambel (1984) who reported highest feed intake in $10 \%$ Azolla meal fed broilers compared to $0,5,10,15,20$ or $25 \%$ Azolla meal fed groups. Seth et al., (2014) also reported numerically higher consumption at $10 \%$ than at $15 \%$ Azolla inclusion level.

The findings in the present study are at variance with those of Querubin et al., (1986) who reported consistent increase in feed intake when Azolla meal was fed at 0, 5, 10, $15 \%$ level.

These differences in the findings between the present study and the other ones might be attributed to chicken types used with varying capacities of fibre degradation along with environmental factors. In the enzyme supplemented groups, in the present study, the 15\% Azolla group (T4) showed higher consumptions than the $10 \%$ group (T2).

These findings are in the line of those reported by Querubin, (1986), Sarria and preston (1995) and 13. Parthasarathy et al., (2002) even though these authors did not use enzyme. Chennegowda et al., (2001) observed that enzyme preparations (xylanase and pectinase or xylanase and cellulase) improved digestibility of NSPs by enzyme supplementation only at sufficient levels of substrate (20\% SFE) as feed enzyme had higher Km. Hence Azolla at $10 \%$ level might have been an adequate substrate for the enzyme action.

In the 15\% Azolla groups the enzyme group (T4) showing higher consumptions than the non-enzyme group (T3) might be attributed to the significantly $(\mathrm{p} \leq 0.05)$ higher body weights (5th and 6th week) attained by the enzyme group (T4) with commensurate higher feed consumptions. Another reason could be that the enzyme level might have been inadequate leading to incomplete fibre degradation resulting in lower release of energy, thereby causing increased feed consumption

\section{Feed conversion ratio (FCR)}

The cumulative weekly FCR values, for the birds in the five groups, are also presented in the Table 7. There was no significant $(\mathrm{p} \geq 0.05)$ difference between the groups in the $2^{\text {nd }}$ week. In the $3^{\text {rd }}$ and $4^{\text {th }}$ weeks, each of the Azolla fed groups showed significantly $(p \leq 0.05)$ lower values than the control.

In the $5^{\text {th }}$ week, the Azolla fed groups except group $\mathrm{T} 1$, showed lower $(\mathrm{p} \leq 0.05)$ values than the control $(\mathrm{C} 1)$, there being no difference $(p \geq 0.05)$ between groups $\mathrm{C} 1$ and $\mathrm{T} 1$. In $6^{\text {th }}$ week, no significant $(\mathrm{p} \leq 0.05)$ difference was noticed between the groups, but in $7^{\text {th }}$ week, significantly $(p \leq 0.05)$ lower values in each of Azolla fed groups, as compared to control were observed.

Between the Azolla fed groups, no significant $(\mathrm{p} \geq 0.05)$ difference was recorded, except in the $3^{\text {rd }}$ week, when group T4 showed a lower value $(p \leq 0.05)$ than group $\mathrm{T} 1$ without any difference $(p \geq 0.05)$ with any of the other two groups. 
Table.1 Ingredient composition of broiler starter and broiler finisher ration

\begin{tabular}{|l|c|c|c|c|c|c|c|c|c|c|}
\hline \multirow{2}{*}{\multicolumn{1}{|c|}{ Ingredients }} & \multicolumn{4}{|c|}{ Broiler Starter } & \multicolumn{5}{c|}{ Broiler Finisher } \\
\cline { 2 - 13 } & $\mathbf{C}_{\mathbf{1}}$ & $\mathbf{T}_{\mathbf{1}}$ & $\mathbf{T}_{\mathbf{2}}$ & $\mathbf{T}_{\mathbf{3}}$ & $\mathbf{T}_{\mathbf{4}}$ & $\mathbf{C}_{\mathbf{1}}$ & $\mathbf{T}_{\mathbf{1}}$ & $\mathbf{T}_{\mathbf{2}}$ & $\mathbf{T}_{\mathbf{3}}$ & $\mathbf{T}_{\mathbf{4}}$ \\
\hline Maize & 55.0 & 50.3 & 50.3 & 46.4 & 46.4 & 60.0 & 57.25 & 57.25 & 42.4 & 42.4 \\
\hline Soya bean Meal & 39.5 & 36.0 & 36.0 & 34.2 & 34.2 & 31.0 & 28.0 & 28.0 & 27.3 & 27.3 \\
\hline Deoiled Rice bran & 2.5 & 0.0 & 0.0 & 0.0 & 0.0 & 5.25 & 2.0 & 2.0 & 0.5 & 0.5 \\
\hline Oil & 0.0 & 1.5 & 1.5 & 2.8 & 2.8 & 0.75 & 1.75 & 1.75 & 3.0 & 3.0 \\
\hline Azolla & 0.0 & 9.2 & 9.2 & 13.6 & 13.6 & 0.0 & 8.0 & 8.0 & 12.0 & 12.0 \\
\hline $\begin{array}{l}\text { Mineral mixture } \\
\text { and Common Salt }\end{array}$ & 3.0 & 3.0 & 3.0 & 3.0 & 3.0 & 3.0 & 3.0 & 3.0 & 3.0 & 3.0 \\
\hline Total & 100 & 100 & 100 & 100 & 100 & 100 & 100 & 100 & 100 & 100 \\
\hline Enzyme (K zyme) & - & - & + & - & + & - & - & + & - & + \\
\hline
\end{tabular}

Table.2 Nutrient composition of experimental broiler starter and broiler finisher ration

\begin{tabular}{|c|c|c|c|c|c|c|}
\hline \multirow{3}{*}{ Nutrient } & \multicolumn{3}{|c|}{ Broiler Starter } & \multicolumn{3}{|c|}{ Broiler Finisher } \\
\hline & $\mathbf{C}_{1}$ & $\mathbf{T}_{1} / \mathbf{T}_{\mathbf{2}}$ & $\Gamma_{3} / \mathbf{T}_{4}$ & $\mathbf{C}_{1}$ & $\mathbf{T}_{1} / \mathbf{T}_{2}$ & $\mathbf{T}_{3} / \mathbf{T}_{\mathbf{4}}$ \\
\hline & \multicolumn{3}{|c|}{ Percentage on dry matter basis } & \multicolumn{3}{|c|}{ Percentage on dry matter basis } \\
\hline Moisture & 11.0 & \begin{tabular}{l|l}
12.2 \\
\end{tabular} & 12.8 & 13.14 & 12.90 & 13.25 \\
\hline Crude protein & 23.17 & 23.15 & 22.78 & 20.14 & 20.12 & 20.01 \\
\hline Ether Extract & 1.05 & 1.42 & 1.60 & 1.57 & 1.78 & 2.03 \\
\hline Crude fibre & 4.01 & 4.63 & 4.92 & 4.03 & 4.30 & 4.89 \\
\hline Total Ash & 6.34 & 7.48 & 7.97 & 6.37 & 7.20 & 7.87 \\
\hline Nitrogen free extract & 65.43 & 63.32 & 62.73 & 67.89 & 66.60 & 65.20 \\
\hline Calcium & 0.92 & 1.08 & 1.14 & 0.82 & 0.91 & 0.91 \\
\hline Available Phosphorus & 0.48 & 0.51 & 0.50 & 0.53 & 0.51 & 0.54 \\
\hline $\begin{array}{l}\text { Metabolisable } \\
\text { energy*(Kcal/kg) }\end{array}$ & 2800.50 & 2809.26 & 2805.00 & 2900.00 & 2902.21 & 2902.00 \\
\hline
\end{tabular}

*Calculated value

Table.3 Calculated feed costs of starter rations

\begin{tabular}{|l|c|c|c|c|c|c|}
\hline \multicolumn{1}{|c|}{ Ingredient } & Cost//kg (Rs) & $\mathbf{C}_{\mathbf{1}}$ & $\mathbf{T}_{\mathbf{1}}$ & $\mathbf{T}_{\mathbf{2}}$ & $\mathbf{T}_{\mathbf{3}}$ & $\mathbf{T}_{\mathbf{4}}$ \\
\hline Maize & 18 & 990 & 905.4 & 905.4 & 835.2 & 835.2 \\
\hline Soya & 45 & 1777.5 & 1620 & 1620 & 1539 & 1539 \\
\hline Dorb & 10 & 25 & 0 & 0 & 0 & 0 \\
\hline Oil & 60 & 0 & 90 & 90 & 168 & 168 \\
\hline Azolla & 1 & 0 & 9.2 & 9.2 & 13.6 & 13.6 \\
\hline Mineral Mix & 60 & 120 & 120 & 120 & 120 & 120 \\
\hline Salt & 10 & 10 & 10 & 10 & 10 & 10 \\
\hline TOTAL/100 Kg & & 2922.5 & 2769.6 & 2769.6 & 2713.8 & 2713.8 \\
\hline Enzyme @ 50g/100kg feed & 300 & 0 & 0 & 15 & 0 & 15 \\
\hline Total cost including enzyme cost & & 2922.5 & 2769.6 & 2784.6 & 2713.8 & 2728.8 \\
\hline Feed cost/kg & & 29.22 & 27.69 & 27.84 & 27.13 & 27.28 \\
\hline
\end{tabular}


Table.4 Calculated feed costs of finisher rations

\begin{tabular}{|l|c|c|c|c|c|c|}
\hline \multicolumn{1}{|c|}{ Ingredient } & $\mathbf{C o s t / ~} \mathbf{k g}(\mathbf{R s})$ & $\mathbf{C}_{\mathbf{1}}$ & $\mathbf{T}_{\mathbf{1}}$ & $\mathbf{T}_{\mathbf{2}}$ & $\mathbf{T}_{\mathbf{3}}$ & $\mathbf{T}_{\mathbf{4}}$ \\
\hline Maize & 18 & 1080 & 1030.5 & 1030.5 & 763.2 & 763.2 \\
\hline Soya & 45 & 1395 & 1260 & 1260 & 1228.5 & 1228.5 \\
\hline Dorb & 10 & 52.5 & 20 & 20 & 5 & 5 \\
\hline Oil & 60 & 45 & 105 & 105 & 180 & 180 \\
\hline Azolla & 1 & 0 & 8 & 8 & 12 & 12 \\
\hline Mineral Mix & 60 & 120 & 120 & 120 & 120 & 120 \\
\hline Salt & 10 & 10 & 10 & 10 & 10 & 10 \\
\hline cost/100 kg & & 2710.0 & 2571.0 & 2571.0 & 2348.7 & 2348.7 \\
\hline Enzyme @ 50g/100kg feed & 300 & 0 & 0 & 15 & 0 & 15 \\
\hline Total cost including enzyme cost & & 2710 & 2571 & 2586 & 2348 & 2363 \\
\hline Feed cost/kg & & 27.10 & 25.71 & 25.86 & 23.48 & 23.63 \\
\hline
\end{tabular}

Table.5 Chemical composition of Azolla meal (D.M basis)

\begin{tabular}{|l|c|c|c|}
\hline Nutrients & Percentage & Nutrients & Percentage \\
\hline Dry matter & 91.07 & Calcium & 1.10 \\
\hline Crude protein & 25.40 & Phosphorus & 0.55 \\
\hline Crude fibre & 14.23 & Zinc $(\mathrm{ppm})$ & 158.6 \\
\hline Ether extract & 2.58 & Copper $(\mathrm{ppm})$ & 7.33 \\
\hline Total ash & 18.76 & Manganese $(\mathrm{ppm})$ & 83.92 \\
\hline NFE & 39.03 & Iron $(\mathrm{ppm})$ & 283.3 \\
\hline
\end{tabular}

Table.6 Average weekly body weight $(\mathrm{g})$ of broilers and absolute gains ( $\mathrm{g}$ ) of broilers

\begin{tabular}{|c|c|c|c|c|c|c|c|c|c|c|c|c|}
\hline \multirow{2}{*}{$\begin{array}{l}\text { Age in } \\
\text { Week }\end{array}$} & \multicolumn{5}{|c|}{ Body Weight(g) } & \multirow{2}{*}{$\begin{array}{c}\mathbf{P} \\
\text { Value }\end{array}$} & \multicolumn{5}{|c|}{ Absolute gains(g) } & \multirow{2}{*}{$\begin{array}{c}P \\
\text { Value }\end{array}$} \\
\hline & $\mathrm{C}_{1}$ & $\mathrm{~T}_{1}$ & $\mathrm{~T}_{2}$ & $\mathrm{~T}_{3}$ & $\mathrm{~T}_{4}$ & & $\mathrm{C}_{1}$ & $\mathrm{~T}_{1}$ & $\mathrm{~T}_{2}$ & $\mathrm{~T}_{3}$ & $\mathrm{~T}_{4}$ & \\
\hline $1^{\text {st }}$ & $\begin{array}{c}64.73 \pm \\
0.58\end{array}$ & $\begin{array}{c}65.80 \pm \\
2.00\end{array}$ & $\begin{array}{c}65.43 \pm \\
0.47\end{array}$ & $\begin{array}{c}64.76 \pm \\
1.21\end{array}$ & $\begin{array}{c}65.56 \pm \\
1.78\end{array}$ & 1.00 & - & - & - & - & - & - \\
\hline $2^{\text {nd }}$ & $\begin{array}{c}97.83 \pm \\
1.76\end{array}$ & $\begin{array}{c}98.43 \pm \\
1.95\end{array}$ & $\begin{array}{c}98.26 \pm \\
2.31\end{array}$ & $\begin{array}{c}97.96 \pm \\
1.68\end{array}$ & $\begin{array}{c}98.40 \pm \\
1.85\end{array}$ & 0.99 & $\begin{array}{c}33.10 \pm \\
0.75\end{array}$ & $\begin{array}{c}32.56 \pm \\
0.51\end{array}$ & $\begin{array}{c}33.33 \pm \\
2.42\end{array}$ & $\begin{array}{c}33.03 \pm \\
1.59\end{array}$ & $\begin{array}{c}33.30 \pm \\
1.36\end{array}$ & 0.99 \\
\hline $3^{\text {rd }}$ & $\begin{array}{c}133.83^{\mathrm{c}} \\
\pm 3.73\end{array}$ & $\begin{array}{c}195.10^{\mathrm{b}} \\
\pm 5.20\end{array}$ & $\begin{array}{c}195.90^{\mathrm{b}} \\
\pm 6.05\end{array}$ & $\begin{array}{c}213.10^{\mathrm{a}} \\
\pm 6.92 \\
\end{array}$ & $\begin{array}{c}228.00^{\mathrm{a}} \\
\pm 6.80\end{array}$ & 0.00 & $\begin{array}{l}69.21^{\mathrm{d}} \\
\pm 3.93 \\
\end{array}$ & $\begin{array}{c}130.40^{\mathrm{c}} \\
\pm 2.51\end{array}$ & $\begin{array}{c}130.97^{\mathrm{C}} \\
\pm 2.18\end{array}$ & $\begin{array}{c}147.67^{b} \\
\pm 4.04 \\
\end{array}$ & $\begin{array}{c}162.90^{\mathrm{a}} \\
\pm 5.49 \\
\end{array}$ & 0.00 \\
\hline $4^{\text {th }}$ & $\begin{array}{l}329.86^{\mathrm{c}} \\
\pm 12.40\end{array}$ & $\begin{array}{c}530.07^{\mathrm{b}} \\
\pm 14.76\end{array}$ & $\begin{array}{l}568.38^{\mathrm{a}} \\
{ }^{\mathrm{b}} \pm 16.32\end{array}$ & $\begin{array}{l}568.72^{\mathrm{a}} \\
{ }^{\mathrm{b}} \pm 17.95\end{array}$ & $\begin{array}{l}612.93^{\mathrm{a}} \\
\pm 17.14\end{array}$ & 0.00 & $\begin{array}{c}265.19 \\
{ }^{\mathrm{c}} \pm 27.9 \\
4\end{array}$ & $\begin{array}{c}464.85^{\mathrm{b}} \\
\pm 9.69\end{array}$ & $\begin{array}{l}504.24^{\mathrm{a}} \\
{ }^{\mathrm{b}} \pm 13.23\end{array}$ & $\begin{array}{l}502.67^{\mathrm{a}} \\
{ }^{\mathrm{b}} \pm 21.48\end{array}$ & $\begin{array}{c}548.02^{\mathrm{a}} \\
\pm 1.45\end{array}$ & 0.00 \\
\hline $5^{\text {th }}$ & $\begin{array}{l}616.00^{\mathrm{c}} \\
\pm 15.17\end{array}$ & $\begin{array}{l}848.97^{\mathrm{b}} \\
\pm 14.48\end{array}$ & $\begin{array}{c}880.32^{\mathrm{b}} \\
\pm 18.50\end{array}$ & $\begin{array}{c}882.17^{\mathrm{b}} \\
\pm 18.08\end{array}$ & $\begin{array}{l}935.82^{\mathrm{a}} \\
\pm 19.22\end{array}$ & 0.00 & $\begin{array}{c}553.06 \\
\mathrm{~b} \pm 28.7 \\
9\end{array}$ & $\begin{array}{l}783.67^{\mathrm{a}} \\
\pm 12.81\end{array}$ & $\begin{array}{l}814.68^{\mathrm{a}} \\
\pm 12.91\end{array}$ & $\begin{array}{l}815.57^{\mathrm{a}} \\
\pm 24.63\end{array}$ & $\begin{array}{l}846.31^{\mathrm{a}} \\
\pm 37.80\end{array}$ & 0.00 \\
\hline $6^{\text {th }}$ & $\begin{array}{l}952.21^{\mathrm{c}} \\
\pm 26.45\end{array}$ & $\begin{array}{l}1131.0^{\mathrm{b}} \\
\pm 25.77\end{array}$ & $\begin{array}{l}1161.0^{\mathrm{b}} \\
\pm 37.17\end{array}$ & $\begin{array}{l}1163.3^{\mathrm{b}} \\
\pm 32.98\end{array}$ & $\begin{array}{c}1237.2^{\mathrm{a}} \\
\pm 34.4\end{array}$ & 0.00 & $\begin{array}{c}891.17 \\
\mathrm{~b} \pm 63.9 \\
7\end{array}$ & $\begin{array}{c}1063^{\mathrm{a}} \pm \\
43.14\end{array}$ & $\begin{array}{l}1095.1^{\mathrm{a}} \\
\pm 14.26\end{array}$ & $\begin{array}{l}1095.4^{\mathrm{a}} \\
\pm 45.52\end{array}$ & $\begin{array}{l}1184.9^{\mathrm{a}} \\
\pm 34.23\end{array}$ & 0.00 \\
\hline $7^{\text {th }}$ & $\begin{array}{l}1215.10 \\
b_{ \pm} \pm 25.36\end{array}$ & $\begin{array}{l}1360.5^{\mathrm{a}} \\
\pm 36.88\end{array}$ & $\begin{array}{l}1379.8^{\mathrm{a}} \\
\pm 36.03\end{array}$ & $\begin{array}{l}1377.7^{\mathrm{a}} \\
\pm 37.03\end{array}$ & $\begin{array}{l}1433.1^{\mathrm{a}} \\
\pm 17.84\end{array}$ & 0.00 & $\begin{array}{r}1154^{\mathrm{b}} \\
\pm 31.69\end{array}$ & $\begin{array}{l}1294.2^{\mathrm{a}} \\
\mathrm{b} \pm 40.27\end{array}$ & $\begin{array}{l}1313.9^{\mathrm{a}} \\
\pm 63.41\end{array}$ & $\begin{array}{l}1314.0^{\mathrm{a}} \\
\pm 17.83\end{array}$ & $\begin{array}{l}1376.8^{\mathrm{a}} \\
\pm 62.10\end{array}$ & 0.065 \\
\hline
\end{tabular}

Values bearing different superscripts in a row differ significantly $(\mathrm{P}<0.05)$ 
Table.7 Cumulative weekly feed consumption (g) and feed conversion ratio (FCR) of broilers

\begin{tabular}{|c|c|c|c|c|c|c|c|c|c|c|c|c|}
\hline \multirow[t]{2}{*}{ Age } & \multicolumn{5}{|c|}{ Feed consumption } & \multirow{2}{*}{$\begin{array}{l}\mathbf{P} \\
\text { Value } \\
\end{array}$} & \multicolumn{5}{|c|}{ FCR } & \multirow{2}{*}{$\begin{array}{l}\mathbf{P} \\
\text { Value } \\
\end{array}$} \\
\hline & $\mathbf{C}_{1}$ & $\mathbf{T}_{1}$ & $\mathbf{T}_{2}$ & $\mathbf{T}_{3}$ & $\mathbf{T}_{4}$ & & $\mathrm{C}_{1}$ & $T_{1}$ & $\mathbf{T}_{2}$ & $\mathbf{T}_{\mathbf{3}}$ & $\mathbf{T}_{4}$ & \\
\hline $\begin{array}{c}2^{\text {nd }} \\
\text { week }\end{array}$ & $\begin{array}{c}157.17^{\mathrm{b}} \\
\pm 1.09\end{array}$ & $\begin{array}{c}160.33^{\mathrm{a}} \\
\pm 0.88\end{array}$ & $\begin{array}{c}160.33^{\mathrm{a}} \\
\pm 0.33\end{array}$ & $\begin{array}{c}160.50^{\mathrm{a}} \\
\pm 0.28\end{array}$ & $\begin{array}{c}160.83^{\mathrm{a}} \\
\pm 0.06\end{array}$ & 0.025 & $\begin{array}{c}4.75 \pm \\
0.07\end{array}$ & $\begin{array}{c}4.77 \pm \\
0.04\end{array}$ & $\begin{array}{c}4.86 \pm \\
0.36\end{array}$ & $\begin{array}{c}4.88 \pm \\
0.23\end{array}$ & $\begin{array}{c}4.84 \pm \\
0.19\end{array}$ & 0.99 \\
\hline $\begin{array}{c}3^{\text {rd }} \\
\text { week }\end{array}$ & $\begin{array}{c}338.20^{\mathrm{d}} \\
\pm 1.67 \\
\end{array}$ & $\begin{array}{c}391.50^{\mathrm{b}} \\
\pm 0.86\end{array}$ & $\begin{array}{c}389.00^{\mathrm{b}} \\
\pm 1.15\end{array}$ & $\begin{array}{c}384.27^{\mathrm{c}} \\
\pm 0.89 \\
\end{array}$ & $\begin{array}{c}403.33^{\mathrm{a}} \\
\pm 1.45 \\
\end{array}$ & 0.00 & $\begin{array}{c}4.92^{\mathrm{a}} \pm \\
0.31\end{array}$ & $\begin{array}{c}3.00^{\mathrm{b}} \pm \\
0.06\end{array}$ & $\begin{array}{c}2.97^{\mathrm{bc}} \pm \\
0.04\end{array}$ & $\begin{array}{c}2.60^{\mathrm{bc}} \pm \\
0.06\end{array}$ & $\begin{array}{c}2.48^{\mathrm{c}} \pm \\
0.08\end{array}$ & 0.00 \\
\hline $\begin{array}{c}4^{\text {th }} \\
\text { week }\end{array}$ & $\begin{array}{c}701.97^{\mathrm{d}} \\
\pm 2.32\end{array}$ & $\begin{array}{c}1021.7^{\mathrm{b}} \\
\pm 1.76\end{array}$ & $\begin{array}{c}1013.3 \\
00^{c} \pm 3.3 \\
3\end{array}$ & $\begin{array}{c}1002.1^{\mathrm{c}} \\
\pm 9.13\end{array}$ & $\begin{array}{c}1037.0^{\mathrm{a}} \\
\pm .3 .60\end{array}$ & 0.00 & $\begin{array}{c}2.70^{\mathrm{a}} \pm \\
0.29\end{array}$ & $\begin{array}{c}2.19^{\mathrm{b}} \pm \\
0.04\end{array}$ & $\begin{array}{c}2.01^{\mathrm{b}} \pm \\
0.04\end{array}$ & $\begin{array}{c}2.00^{\mathrm{b}} \pm \\
0.10\end{array}$ & $\begin{array}{c}1.89^{\mathrm{b}} \pm \\
0.00\end{array}$ & 0.01 \\
\hline $\begin{array}{c}5^{\text {th }} \\
\text { week }\end{array}$ & $\begin{array}{l}1315.5^{\mathrm{d}} \\
\pm 26.42 \\
\end{array}$ & $\begin{array}{c}1744.1^{\mathrm{b}} \\
\pm 2.83\end{array}$ & $\begin{array}{c}1669.5^{\mathrm{c}} \\
\pm 1.75 \\
\end{array}$ & $\begin{array}{c}1704.3^{\mathrm{c}} \\
\pm 3.12\end{array}$ & $\begin{array}{c}1799.8^{\mathrm{a}} \\
\pm 2.16 \\
\end{array}$ & 0.00 & $\begin{array}{c}2.38^{\mathrm{a}} \pm \\
0.09\end{array}$ & $\begin{array}{l}2.22^{\mathrm{ab}} \\
\pm 0.03 \\
\end{array}$ & $\begin{array}{c}2.05^{\mathrm{b}} \pm \\
0.03\end{array}$ & $\begin{array}{c}2.09^{\mathrm{b}} \pm . \\
06\end{array}$ & $\begin{array}{c}2.13^{\mathrm{b}} \pm \\
0.09\end{array}$ & 0.04 \\
\hline $\begin{array}{c}6^{\text {th }} \\
\text { week } \\
\end{array}$ & $\begin{array}{l}2146.7^{\mathrm{c}} \\
\pm 64.37 \\
\end{array}$ & $\begin{array}{c}2476.7^{\mathrm{b}} \\
\pm 3.33\end{array}$ & $\begin{array}{l}2385.2^{\mathrm{b}} \\
\pm 17.63 \\
\end{array}$ & $\begin{array}{c}2445.2^{\mathrm{b}} \\
\pm 4.46\end{array}$ & $\begin{array}{c}2582.0^{\mathrm{a}} \\
\pm 9.16 \\
\end{array}$ & 0.00 & $\begin{array}{c}2.42 \pm \\
0.11 \\
\end{array}$ & $\begin{array}{c}2.33 \pm \\
0.09 \\
\end{array}$ & $\begin{array}{c}2.17 \pm \\
0.04\end{array}$ & $\begin{array}{c}2.24 \pm \\
0.09 \\
\end{array}$ & $\begin{array}{c}2.18 \pm \\
0.06\end{array}$ & 0.25 \\
\hline $\begin{array}{c}7^{\text {th }} \\
\text { week }\end{array}$ & $\begin{array}{l}3023.3^{\mathrm{c}} \\
\pm 92.43\end{array}$ & $\begin{array}{c}3270.0 . \\
0^{\mathrm{a}} \pm 10.0 \\
0\end{array}$ & $\begin{array}{l}3160.0^{\mathrm{b}} \\
\pm 10.00\end{array}$ & $\begin{array}{l}3217.0^{\mathrm{b}} \\
\pm 12.99\end{array}$ & $\begin{array}{l}3396.7^{\mathrm{a}} \\
\pm 16.66\end{array}$ & 0.001 & $\begin{array}{c}2.61^{\mathrm{a}} \pm \\
0.01\end{array}$ & $\begin{array}{c}2.53^{\mathrm{b}} \pm \\
0.08\end{array}$ & $\begin{array}{c}2.41^{\mathrm{b}} \pm \\
0.11\end{array}$ & $\begin{array}{c}2.44^{\mathrm{b}} \pm \\
0.02\end{array}$ & $\begin{array}{c}2.47^{\mathrm{b}} \pm \\
0.09\end{array}$ & 0.04 \\
\hline
\end{tabular}

Values bearing different superscripts in a row differ significantly $(\mathrm{P}<0.05)$

Table.8 Economics of broiler production

\begin{tabular}{|c|c|c|c|c|c|c|}
\hline \multirow{2}{*}{ Economics } & \multirow{2}{*}{ Components } & \multicolumn{5}{|c|}{ Groups } \\
\hline & & $\mathrm{C}_{1}$ & $T_{1}$ & $\mathbf{T}_{2}$ & $T_{3}$ & $\mathbf{T}_{4}$ \\
\hline \multirow{7}{*}{$\begin{array}{l}\text { Expenditure } \\
\text { /per bird }\end{array}$} & $\begin{array}{llll}\text { a. } & \begin{array}{l}\text { Cost of starter ration } \\
(\mathrm{Rs} / \mathrm{kg})\end{array}\end{array}$ & 29.22 & 27.69 & 27.84 & 27.13 & 27.28 \\
\hline & b. $\begin{array}{l}\text { Cost of finisher ration } \\
(\mathrm{Rs} / \mathrm{kg})\end{array}$ & 27.10 & 25.71 & 25.86 & 23.48 & 23.63 \\
\hline & $\begin{array}{l}\text { c. Feed consumed in starter } \\
\text { phase }(\mathrm{g})\end{array}$ & 428.20 & 481.50 & 479.18 & 474.26 & 493.33 \\
\hline & $\begin{array}{l}\text { d. Feed consumed in finisher } \\
\text { phase }(\mathrm{g})\end{array}$ & 2685.13 & 2878.5 & 2771.0 & 2833.4 & 2993.33 \\
\hline & $\begin{array}{ll}\text { e. Feed cost in starter phase } \\
\text { (Rs) }\end{array}$ & 12.51 & 13.33 & 13.33 & 12.87 & 13.46 \\
\hline & $\begin{array}{ll}\text { f. } & \text { Feed cost in finisher phase } \\
& (\mathrm{Rs})\end{array}$ & 72.76 & 74.00 & 71.65 & 66.54 & 70.75 \\
\hline & g. $\quad$ Total feed cost $(\mathrm{Rs})(\mathrm{e}+\mathrm{f})$ & 85.28 & 87.34 & 84.99 & 79.41 & 84.21 \\
\hline \multirow{4}{*}{$\begin{array}{l}\text { Return } \\
\text { /bird }\end{array}$} & h. Live weight $(\mathrm{g})$ in 7 weeks & 1215.10 & 1360.50 & 1379.80 & 1377.70 & 1433.10 \\
\hline & $\begin{array}{ll}\text { i. } & \text { Receipt / bird @ Rs 78/kg } \\
& \text { live weight (Rs) }\end{array}$ & 94.77 & 106.11 & 107.62 & 107.46 & 11.78 \\
\hline & $\begin{array}{ll}\text { j. } & \begin{array}{l}\text { Net return / bird }(\mathrm{i}-\mathrm{g}) \\
(\mathrm{Rs})\end{array}\end{array}$ & 9.49 & 18.77 & 22.62 & 28.04 & 27.56 \\
\hline & $\begin{array}{ll}\text { k. } & \begin{array}{l}\text { Difference in net return } \\
\text { over control }\end{array}\end{array}$ & 0 & 9.28 & 13.13 & 18.54 & 18.06 \\
\hline \multirow{4}{*}{$\begin{array}{l}\text { Return } / \mathrm{kg} \\
\text { live weight }\end{array}$} & $\begin{array}{ll}\text { 1. } & \text { Cost of feed consumed } \\
\text { (Rs) }\end{array}$ & 70.18 & 64.19 & 61.60 & 57.64 & 58.76 \\
\hline & $\begin{array}{ll}\text { m. } & \text { Receipt per kg live wt. } \\
\text { (Rs) }\end{array}$ & 78 & 78 & 78 & 78 & 78 \\
\hline & $\begin{array}{l}\text { n. Net return/ kg live weight } \\
(\mathrm{Rs})(\mathrm{m}-\mathrm{l})\end{array}$ & 7.82 & 13.81 & 16.40 & 20.36 & 19.24 \\
\hline & $\begin{array}{l}\text { o. difference in net return } \\
\text { over control }\end{array}$ & 0 & 5.99 & 8.59 & 12.54 & 11.42 \\
\hline
\end{tabular}


The beneficial effects of dietary Azolla on FCR, as observed in the present study, corroborate those reported by several authors Basak et al., (2002) found increased efficiency at 10\%, (Dhumal et al., 2009) at 2.5 to $5 \%$ level, (Seth et al., 2014) at 5 to $15 \%$ level, and (Chichilichi et al., 2014) at 5\% levels of Azolla in the diet of chicken. Enzyme supplementation in the present study on $10 \%$ Azolla inclusion resulted in nonsignificantly $(\mathrm{p} \geq 0.05)$ better feed efficiency, while on 15\% Azolla, there was no difference. These findings corroborate those of Chichilichi et al., (2014) who found nonsignificant $(\mathrm{p} \geq 0.05)$ better efficiency upon supplementation of enzyme in diets containing 0, 5 or $10 \%$ Azolla. Several studies suggest beneficial effects of enzyme supplementation on feed efficiency (Rajeshwara and and Devegowda., 1996), (Choct et al., 1995) and (Saxena et al., 2006)

\section{Economics of production}

The economics of production, for the birds in different groups, are presented in the Table 8. Each of Azolla fed groups showed higher economic efficiency than the control. Between the Azolla fed groups, group T3 showed the highest efficiency followed by groups T4, T2 and T1, in that order. The higher efficiency shown by the Azolla fed groups were due to greater feed efficiency and weight gain because of feeding Azolla.

Economisation of feed cost in poultry by dietary inclusion of Azolla at different levels has been reported by several authors (Subudhi and Singh 1978) reported that feed cost could be economised by replacing 20-25\% of commercial feed by fresh Azolla. Seth et al., (2014) recorded lowest feed cost for the $15 \%$ Azolla by incorporating $0,5,10$ or $15 \%$ Azolla in the diet of vanaraja birds. But Basak et al., (2002) reported that 5\% Azolla fed group had the highest profit/bird as compared to 0,10 or
15\% Azolla fed groups. Chichilichi et al., (2014) also reported highest profit margin at $5 \%$ level of Azolla inclusion compared to 0 or $10 \%$ level. Naghshi et al., (2014) also reported that the lowest feed cost $/ \mathrm{kg}$ body weight was for diets containing 5\% Azolla in comparison to 10 or $15 \%$ inclusion. The differences observed between the present study and reported works could be attributed to chicken types, feed and environmental condition in these experiment.

From above discussion, it was concluded that Azolla could be included in the broiler ration up to $15 \%$ level which resulted in higher body weight gain and feed efficiency. It leads to substantial economisation of feed cost of production.

\section{Acknowledgments}

The authors acknowledged the Dean, College of Veterinary Science and A.H, Orissa University of Agriculture and Technology, Bhubaneswar. Department of Livestock Production \& Management, Officer in-charge, Instructional Livestock Farm. Department of Livestock Products and Technology providing necessary facility and support to carry out this research work.

\section{References}

A.O.A.C. (1995) Official methods of analysis, Association of Official Analytical Chemists, 13th edn. Washington DC.

Alalade, O.A. and Iyayi, E.A. (2006) Chemical composition and feeding value of Azolla (Azolla pinnata) meal for egg type chicks. Int. J. Poult. Sci., 5: 137-41

Alalade, O.A., Eustace, A., Iyayi, E.A., Taiwo, O. and Alalade. (2007) The nutritive value of Azolla (Azolla pinnata) meal in diets for growing pullets and subsequent effect on laying performance. J.Poult.Sci., 44:273-277

Alcantara, P. F. and Querubin, L.J. (1985) Feeding value of Azolla meal for broilers. Philippines J. Vet. and Anim. Sci., 11: 1-8 
Balaji, K., Jalaludeen, A., Churchil, R. R., Peethambaran, P.A. and Senthilkumar, S. (2009) Effect of dietary inclusion of Azolla (Azolla pinnata) on production performance of broiler chicken. Indian J. Poult. Sci., 44 (2):195-198

Basak, B., Pramanik, M.A.H., Rahman, M.H., Tarafdar, S.U. and Roy, B.C. (2002) Azolla (Azolla pinnata) as a feed ingredient in broiler ration. Int. J. Poult. Sci., 1: 29-34.

Becerra, M., Preston, T.R. and Ogle, B. (1995) Effect of replacing whole boiled soya beans with Azolla in the diets of growing ducks. Livest. Res. Rural Dev., 7: 1-11.

Bedford, M.R. (1997) Factors affecting response of wheat based diets to enzyme supplementation. Recent Advances in Animal Nutrition conference proceedings. Pp: 1-7.

Bhuiyan, M. A. H., Hasanat, M. R., Ali, M. A. and Rahman, M. A. (1998) Effect of feeding Azolla (Azolla pinnata) on the performance of broiler. Bangladesh J. Anim. Sci., 27(1\&2): 77-82

Broz, J., Oldale, P. and Perrin-Voltz, A.H. (1994) Effects of Trichoderma viride enzyme complex in broiler chickens. Archiv Geflügelkd 58: 130-134.

Buckingham, K.W., Ela, S.W., Morris, J.G. and Goldman, C.R. (1978) Nutritive value of nitrogen-fixing aquatic fern Azolla filiculoides. J. Agric. Food Chem. 26: 12301234.

Cambel, I.M. (1984) Growth performance of broilers fed with varying levels of Azolla meal. Thesis submitted in University of Southern Mindanao, Kabacan, North Cotabato.

Cambel, I.M. (1984). Growth performance of broilers fed with varying levels of Azolla meal. Thesis submitted in University of Southern Mindanao, Kabacan, North Cotabato.

Chennegowda, K. K., Gowda. and Devegowda, G. (2001) Efficacy of enzyme mixture to improve performance of broilers fed sunflower extraction rich diets. Indian $\mathrm{J}$. Poult. Sci., 35 (3): 256-259.

Cherryl, D.M., Prasad, R.M.V., Jagadeeswararao, S., Jayalaxmi, P. and Kumar, D.S. (2014) A study on the nutritive value of Azolla pinnata. Livest. Res. Int., 2: 13-15.
Chichilichi, B. (2014) Protein substitution with Azolla on growth and carcass characteristic of commercial broiler chicken. M.V.Sc Thesis submitted to the Orissa University of Agriculture and Technology, Bhubaneswar

Choct, M., Hughes, R. J., Tremble, R.P., Angkanaporn, K. and Annison, G. (1995) Non-starch polysaccharide degrading enzymes increase the performance of broiler chickens fed wheat of low apparent metabolizable energy. J. Nutri. 125 (3): 485492

Dhumal, M.V., Siddiqui, M.F., Siddiqui, M.B.A. and Avari, P. E. (2009) Performance of broilers fed on different levels of Azolla meal. Indian J. Poult. Sci., 44 (1):65-68.

Gunal, M. and Yasar, S. (2004) Performance and some digesta parameters of broiler chickens given low or high viscosity wheat-based diets with or without enzyme supplementation. Turk. J. Vet. Anim. Sci., 28: 323-327

Haustein, A. T., Gilman, R. H., Skillicorn, P. W., Hannan, H., Díaz, F., Guevara, V., Vergara, V., Gastañaduy, A. and Gilman, J.B. (1994) Performance of broiler chickens fed diets containing duckweed (Lemna gibba). J. Agric. Sci., 122 (2):285-289.

ICRA. (2013) Investment Information and Credit Rating Agency of India Ltd., Moddy's Investment Company India Pvt. Ltd., Kolkata-70020 (www.icra.in).

Isshiki, Y. and Nakahiro, Y. (1989) Feed digestibility in different intestinal parts of chicken. Jap. J. Zootech. Sci., 60: 1082-86.

Lawas, M.V.P., Roxas, D.B. and Lambio, A.L. (1998) Laying performance of Philippine Mallard ducks fed diets substituted with fresh Azolla. Recent Developments in Animal Production- 1998: proceedings of the Philippine Society of Animal Science 35th Annual Convention, p. 220-225.

Naghshi, H., Khojasteh, S., Jafari, M. (2014) Investigation the Effect of Different Levels of Azolla (Azolla pinnata) on Performance and Carcass Characteristics of Cobb Broiler Chicks Int. J. Farming Allied Sci., 3(1):45-49

Namra, M. M. M., Hataba, N. A. and Abdel, W, M. (2010) The productive performance of growing fayoumi chicks fed restricted diets supplemented with free fresh Azolla. Egypt. Poult. Sci., 30 (III): 747-762. 
Onu, P.N., Madubuike, F.N., Onu, D.O. and Ekenyem, B.U. (2011) Performance and economic analysis of broiler starter chicks fed enzyme supplemented sheep manure-based diets. ARPN J. Agric. Biol Sci., 6: 14-1.

Parashuramulu, S. and Nagalakshmi, D. (2012) Azolla - A potential protein supplement for livestock. Livest. Line., 6 (4):18-21.

Parthasarathy, R., Kadrivel, R. and Kathaperumal, V. (2002) Azolla as a partial replacement for fishmeal in boiler rations. Indian Vet. J., 79:144-146.

Pillai, P. K., Premalatha, S. and Rajamony, S. (2002) Azolla- A sustainable feed substitute for livestock. Leisa India., 21(3): 15-17.

Ponnuvel, P., Jalaludeen, A. and Ramakrishnan, A. (2001) Influence of cellulase enzyme supplementation in high fibre diet on the production performance of layer chicken. Indian J. Poult. Sci,. 36 (1): 98-105.

Querubin, L.D., Alcantara, P.F., Luis, E.S. and Princesa, A.O. (1986) Chemical composition and feeding value of Azolla in broiler ration [Philippines]. Philippines J. Vet. and Anim. Sci., 12(3-4): 65.

Rajeshwara rao, N. and Devegowda, G. (1996) Study on dietary supplementation of enzymes on broiler performance. In: Proc. XX World's Poult. Conf., New Delhi, pp 218.

Samarsinghe, K., Messikommer, R. and Wenk, C. (2000) Activity of supplemental, enzymes and their effect on nutrient utilisation and growth performance of growing chickens as affected by pelleting temperature. Arc. Anim. Nutr., 53: 45 - 58.

Sarria, P. and Preston, T. R. (1995) A chick assay method for the evaluation of nonconventional protein sources derived from nacedero (Trichanthera gigantea) and Azolla (Azolla filiculoides). Livest. Res. Rural Dev., 7 (3):6

Saxena, M. J., Karla, A. and Sharma, M. (2006) Feed fortification- Drivers for the decade In: Proc. of VI Biennial Conf. of Anim. Nutr. Assoc., Sep 15th -17th, Jammu, India, pp 89.

Seth, N. (2014) Performance of Banaraja Chicken fed fresh Azolla as protein substitute. M.V.Sc Thesis submitted to the Orissa university of Agriculture and Technology, Bhubaneswar.

Shamna, T.P., Peethambaran, P.A., Jalaludeen, A., Joseph, L. and Aslam, M.K.M. (2013) Broiler characteristics of Japanese quails (Coturnix coturnix japonica) at different levels of diet substitution with Azolla pinnata. Anim. Sci. Rep., 7(2): 75-80

Sittigaipong, R. (1996) Influence of Azolla pinnata on growth performance and carcass quality in broiler. King Mongkut's Agricultural Journal 14(1): 12-20.

Snedecor, G.W. and Cochran, W.G. (1998) Statistical Methods, 6th ed. Oxford and IBH Publishing Company, Kolkata, India.

Subudhi, B. P. R. and Singh, P. K. (1978) Nutritive Value of water fern Azolla pinnata for chicks. Sci. Cult., 44: 234-235.

Talapatra, S.K., Ray, S.C. and Sen, K.C. (1940) Estimation of phosphorus, chlorine, calcium, mgnesium, sodium and potassium in foodstuffs. Indian J. Vet. Sci. Anim. Husbandry.,10: 243-246

Zang, S., Guokia, Zhang B. and Li, T. (1996) Poult. Abstr., 22(3):107.

Zobell, B. I., Choct, M and Freed, R. D. (2000) Intuition of nutrient digestion by wheat Pentosans. Br. J. Nutr., 67: 123-132

\section{How to cite this article:}

Subhranshu Sekhar Rout, C.R. Pradhan, S.K. Mishra, P.K. Pati and Bagh J. 2017. Performance of Coloured Synthetic Broiler Chicken Fed Dried Azolla as Protein Substitute. Int.J.Curr.Microbiol.App.Sci. 6(12): 2349-2358. doi: https://doi.org/10.20546/ijcmas.2017.612.271 